staged as 1A tumours, $42(46.7 \%)$ were upstaged to IB1 $(86 \% \leq 2 \mathrm{~cm}, 14 \% 2-4 \mathrm{~cm}, 0 \%>4 \mathrm{~cm}) ; 76.3 \%$ had conisation as diagnostic procedure. Fourteen out of 547 preoperatively IB1 tumours (2.6\%) were upstaged to IB2 $>4 \mathrm{~cm}$. Analogously 33 patients (6\%) with IB tumours were downstaged to IA. Preoperatively unrecognized parametrial involvement was found by pathology only in 22 out of 637 patients (3.5\%). EUS and MRI were used equally in the study $(53.5 \%$ vs $56.1 \%$ ), both were comparable in the accuracy of tumour size measurement $(2 \mathrm{~cm}$ size categories shift in stage IB) $(p=1.000)$ and in the failure to detect parametrial involvement $(2.9 \%$ vs $4.0 \%)(p=0.535)$. Chart 1 .

Conclusion* Clinical staging with EUS and MRI failed to detect positive parametria only in $3.5 \%$ of patients in the Sentix trial. Upstaging from IA tumours was frequent, mostly after previous conization. Only $2.6 \%$ of patients were upstaged to IB2 tumours $>4 \mathrm{~cm}$ (IB3 FIGO 2018). Both EUS and MRI were equally reliable in tumour size and parametrial involvement assessment.

\section{SENTINEL LYMPH-NODE BIOPSY IN EARLY-STAGE CERVICAL CANCER: THE 4-YEAR FOLLOW-UP RESULTS OF THE SENTICOL 2 TRIAL}

\begin{abstract}
1;2 G Favre*, 1;2B Guani, ${ }^{1} \mathrm{~V}$ Balaya, ${ }^{3} \mathrm{~L}$ Magaud, ${ }^{4} \mathrm{~F}$ Lecuru, 1;2P Mathevet. 'Lausanne University Hospital, Women Mother Child, Lausanne, Switzerland; ${ }^{2}$ University of Lausanne, Faculty of Biology and Medicine, Lausanne, Switzerland; ' ${ }^{3}$ Faculty of Medicine, University of Lyon, Claude Bernard Lyon 1, Clinical Research and Epidemiology Department, Lyon, France; ${ }^{4}$ Curie Institute, Breast, Gynecology and Reconstructive Surgery Unit, Paris, France
\end{abstract}

\subsection{6/ijgc-2021-ESGO.84}

Introduction/Background* Senticol 2 is a randomized multicenter trial in the treatment of early-stage cervical cancer patients. The aim of the Senticol 2 study was to compare the effect of sentinel-lymph-node biopsy (SLNB) to that of SLNB + pelvic lymphadenectomy (PLND), and to determine the postoperative lymphati

Methodology In the Senticol 2 trial, patients underwent a laparoscopy with a sentinel-node-detection procedure and were randomized into two groups, namely: Group A, in which participants received SLNB, and Group B, in which participants received SLNB + PLND. Patients with an intra-operative macroscopically suspicious lymph node, were given a frozen-section evaluation and were randomized only if the results were negative. All of the patients received follow up with a clinical examination at 1,3 , and 6 months after surgery, and then every 3-4 months after that. The median follow up was 51 months (4 years and 3 months).

Result(s)* Disease-free survival after 4 years for the SLNB group and the SLNB + PLND group were $89.51 \%$ and $93.1 \%(p=0.53)$, respectively. The only statistical factor associated with recurrence in the univariate analysis was the adjuvant radiotherapy. No other factors, including the age of the patients, histological type, tumor size, lymph vascular space invasion (LVSI), and positive nodal status, were significant in the univariate or multivariate analyses. The overall survival rates after 4 years in the SLNB and SLNB + PLND groups were $95.2 \%$ and 96\% $(p=0.97)$, with five and four deaths, respectively. The univariate and multivariate analyses did not find any prognostic factors.

Conclusion* This randomized study confirmed the results of the Senticol 1 study and supports the sentinel lymph node (SLN) technique as a safe technique for use in patients with early-stage cervical cancer treated with SLNB only. Diseasefree survival after 4 years was similar in patients treated with SLN biopsy and patients who underwent a lymphadenectomy.

\section{INFLUENCE OF NEW FIGO 2018 STAGING AND TREATMENT OUTCOMES IN EARLY STAGE CERVICAL CANCER: A SINGLE CENTRE STUDY}

${ }^{1} \mathrm{~K}$ Satinder*, ${ }^{1} \mathrm{H}$ Garg, ${ }^{1} \mathrm{M}$ Nandwani, ${ }^{2} \mathrm{M}$ Kalita, ${ }^{1} \mathrm{~S}$ Bansal, ${ }^{1} \mathrm{R}$ Singh. ${ }^{1}$ Dharamshila Narayana Superspeciality Hospital, Delhi, India; ${ }^{2} \operatorname{Dr} B$ Borooah Cancer Institute, Guwahati, India

\subsection{6/ijgc-2021-ESG0.85}

Introduction/Background* Cancer of the cervix is one of the most common cancers in women worldwide. According to GLOBOCAN data of 2020, there were 604,127 new cases of cervical cancer and 341,831 deaths due to the same. This shows that the burden of cervical cancer is still high and further research and implementation of screening programs can help us eliminate this cancer from the world. In our study we have evaluated patients with early-stage cervical cancer who had been treated by surgery followed by risk based tailored adjuvant therapy in our Centre. The clinicopathological features and survival outcomes of these patients were evaluated. These patients were restaged as per new FIGO 2018 staging and its impact on survival evaluated.

Methodology A retrospective study conducted from $1^{\text {st }}$ June 2013 to $31^{\text {st }}$ May 2018 in a tertiary care hospital in North India. All patients of early stage (1B1 to IIA1) cervical cancer who underwent primary surgery followed by tailored adjuvant therapy were analyzed. The overall survival and relapse free survival were calculated. On the basis of histopathology reports, reclassification was done into new FIGO 2018 stage. The survival outcome of both groups was then calculated and compared.

Result(s)* 100 patients of early-stage cancer cervix were studied. All patients underwent open radical hysterectomy with bilateral pelvic lymph node dissection. The median age of the study population was 52.5 years. With a median follow up of 62.1 months the overall survival and relapse free survival was $87.5 \%$ and $92.3 \%$ respectively. The study population was then reclassified according to new FIGO 2018 staging. It was seen that the patients with stage IB1and IB2 cervical cancer had overall survival of $91.1 \%$ and $90 \%$ respectively. The overall survival of stage IB3 was $80 \%$ and the survival of stage IIIC1 was only 60\%.

Conclusion* The new FIGO 2018 staging classification has a significant effect on survival outcome when lymph nodes are involved and also in prognostication of patients with cancer cervix. Surgery followed by risk based appropriate adjuvant therapy is able to provide favorable overall and relapse free survival.

\section{THE OUTCOME OF HIGH-DOSE RATE INTRA-CAVITY BRACHYTHERAPY AND INTENSITY-MODULATED RADIATION THERAPY WITH CENTRAL-SHIELDING FOR CERVICAL CANCER}

1;2Y Mukai*. 'Y Yokohama City University graduate school of Medicine, Radiation Oncology, Japan; '2Shonan Kamakura General Hospital , Radiation Oncology, Japan

10.1136/ijgc-2021-ESG0.86 
Introduction/Background* The high-dose-rate intra-cavity brachytherapy (HDR-ICBT) has an important and essential role for radiation therapy for uterus cervical cancer ad rectal dose reduction is critical for determining the radiation dose for HDR-ICBT. In Asia, standard external beam radiation therapy combines whole pelvic radiation therapy (WPRT) and sequential WPRT with central shielding (WPRT-CS) for rectal dose reduction prior to HDR-ICBT. This study aimed to evaluate the treatment outcome and tolerance of the combination treatment strategy.

Methodology Forty-six cervical cancer patients treated from 8/ 2011 to 9/2020 were included in this retrospective analysis. All patients received combination therapy of HDR-ICBT and EBRT which is consisted of WPRT and WPRT-CS using IMRT/TomoTherapy.

Result(s)* Primary tumor stages (FIGO) were distributed as follows: IB: 6, IIA: 4, IIB: 12, IIIA: 1, IIIB: 3, IIIC1:17, IIIC2:3 (with Para-aortic lymph node metastasis), pelvic lymph node metastasis $(\mathrm{N}+)$ : 21. Forty patients had squamous cell carcinoma (SCC) and the remaining six had adenocarcinoma. The median age of patients was 65.5 (range, 30-91) years. All patients completed treatment, and the median dose of WPRT and WPRT-CS was 36 (range, 20-43.2) and 14.4 (range, 7.228) Gy, respectively. Median HDR-ICBT dose/fractions to Point A was 25 Gy/5 Fr (range, 15-30 Gy/3-5 Fr). Thirty-five had weekly cisplatin concomitant with RT.

Median follow-up was 43.5 (range, 4-122) months, and 5 -year local control, disease-free survival, and overall survival rates (OS) were 95.6\%, 84.0\%, and 83.1\%, respectively. There was only two local failure and seven patients experienced distant metastasis (4 lung metastasis and 3 para-aortic metastasis). Four of seven patients with distant metastasis received salvage therapy and the disease got controlled. One patient who controlled cervical cancer, developed primary lung cancer and died of that. No acute or late $\geq$ Grade 3 radiation-induced genitourinary or gastrointestinal toxicities were observed.

Conclusion* In the present study, the combination of IMRT consisting of WPRT and WPRT-CS using TomoTherapy and HDR-ICBT show comparatively long-term great local disease control. and tolerance rates for cervical cancer patients. This combination strategy is considered one of the effective and safe treatment options for cervical cancer.

\section{FALSE NEGATIVE RATE AT 18F-FDG PET/CT INPARA- AORTIC LYMPHNODE INVOLVEMENT INPATIENTS WITH LOCALLY ADVANCED CERVICALCANCER: IMPACT OF PET TECHNOLOGY}

S Gouy*, V Seebacher, C Chargari, M Terroir, A Maulard, C Genestie, P Pautier, A Leary, P Morice, D Deandreis. Gustave Roussy, Surgery, Villejuif

\subsection{6/ijgc-2021-ESGO.87}

Introduction/Background* The identification of factors responsible for false negative $(\mathrm{FN})$ rate at $18 \mathrm{~F}$ - Fluorodeoxyglucose (FDG) Positron Emission Tomography/Computed Tomography (PET/CT) in para-aortic (PA) lymph nodes in the presurgical staging of patients with locally advanced cervical cancer (LACC) is challenging. The aim of this study was to evaluate the impact of PET/CT technology.

Methodology A total of 240 consecutive patients with LACC (International Federation of Gynecology and Obstetrics, FIGO, stage IB2-IVA) and negative Magnetic Resonance Imaging (MRI) and/or Computed Tomography (CT) and negative 18FFDG PET/CT in the PA region, undergoing laparoscopic PA lymphadenectomy before chemoradiotherapy were included. The FN rate in patients studied with Time of flight (TOF) PET/CT (TOF PET) or non-Time of flight PET/CT (no-TOF PET) technology was retrospectively compared.

Result(s)* Patients presented with FIGO stage IB ( $\mathrm{n}=78)$, stage IIA-B $(\mathrm{n}=134)$, stage III $(\mathrm{n}=18)$ and stage IVa $(\mathrm{n}=$ $10)$, squamous cell carcinoma $(\mathrm{n}=191)$ and adenocarcinoma ( $\mathrm{n}=49$ ). 141/240 patients were evaluated with no-TOF PET/ CT and 99/240 with TOF PET/CT. Twenty-two patients (9\%) had PA nodal involvement at histological analysis and considered PET/CT FN findings. The FN rate was $8.5 \%$ for noTOF PET and $10 \%$ for TOF PET subgroup respectively ( $\mathrm{p}=$ 0.98). Ninety patients (38\%) presented with pelvic node uptakes at PET/CT. The FN rate in the PA region was $18 \%$ $(16 / 90)$ and $4 \%(6 / 150)$ in patients with and without pelvic node involvement at PET/CT respectively (19 vs 3\% for noTOF PET and 17 vs 5\% for TOF PET subgroup).

Conclusion* In LACC, FN rate in PA lymph nodes detection is a clinical issue even for modern PET/CT, especially in patients with pelvic uptake. Surgical lymphadenectomy should be performed in case of negative PET/CT at PA level in these patients, while it could be discussed in the absence of pelvic uptake.

\section{A NEW SURGICAL ERA FOLLOWING THE LACC TRIAL - A TEN STEP SURGICAL APPROACH TO FERTILITY SPARING ABDOMINAL RADICAL TRACHELECTOMY}

S Smyth, H Jiang* , C Pappa, M Abdalla, H Soleymani. John Radcliffe Hospital, UK

\subsection{6/ijgc-2021-ESG0.88}

Introduction/Background* Minimally invasive surgery in the management of cervical cancer was dealt a heavy blow in 2018, with the laparoscopic approach to cervical cancer trial (LACC) taking the gynaecology oncology world by storm; leading to a complete overhaul of surgical techniques involved.

Methodology We present a 34-year-old fit and well nulliparous female referred with grade two squamous cell carcinoma; $4.3 \mathrm{~mm}$ invasion depth on loop histology, stage 1A2. There was no radiological evidence of residual tumour, lymphadenopathy or metastatic disease. Following counsel, the patient underwent midline laparotomy, bilateral pelvic lymph node dissection and radical trachelectomy with no residual and 37 lymph nodes negative and uncomplicated recovery.

Result(s)* We present a stepwise surgical approach:

1. Midline laparotomy and pelvic side wall opening to develop eight avascular retroperitoneal spaces of pelvis with slinging of round ligaments and ureters and preservation of fallopian tubes and ovaries

2. Bilateral pelvic lymph node dissection sent for frozen section examination

3. Full mobilisation of cervix with ureterolysis, dissection of parametrium, paracolpium and vaginal cuff. Skeletonisation to bifurcation, sacrifice and preservation of descending and ascending uterine artery branches respectively - ensuring artery pulsation and uterine body perfusion during procedure

4. Measurement of the vaginal cuff from the central tumour bed - identification of a $2 \mathrm{~cm}$ clear margin 